\title{
Mixing Flow Characteristics for a Transverse Sonic Jet Injecting into a Supersonic Crossflow
}

\author{
El-Hadi Khali ${ }^{1}$ and Yufeng $\mathrm{Yao}^{2}$ \\ ${ }^{I}$ Institute of Aeronautics and Space Studies, Saad Dahleb University at Blida, Blida 09000, Algeria \\ ${ }^{2}$ Department of Engineering Design and Mathematics, University of the West of England, Bristol BS16 1QY, UK
}

\begin{abstract}
The mixing flow characteristics resulted from the interactions between a sonic jet issuing perpendicularly into a supersonic crossflow are studied by using computational fluid dynamics (CFD) approach that applies hybrid Reynolds-averaged Navier-Stokes (RANS) and large-eddy simulation (LES) on a multi-block structured grid, together with a fully implicit time integration, and a low-dissipation flux evaluation scheme. The adopted approach allows for the simulations to resolve the unsteady large-scale structures that play an important role in the mixing process, for which steady RANS simulations often have the difficulties to accurately capture the details. The purpose of this work is to validate the hybrid RANS-LES simulation results against available experimental measurements and to explore its further capabilities in predicting the mixing flow phenomena. The supersonic boundary layer flow considers conditions of an incoming Mach number 1.6 and a Reynolds number $\operatorname{Re}_{\delta}=1.08 \times 10^{5}$, based on the free-stream quantities and the boundary layer thickness upstream of the jet exit in absence of the jet flow. Both the geometry configuration and the flow conditions are taken from a previous experimental study. The simulated results are compared to the experiment, including the mean and the standard deviation of the passive scalar and velocity component profiles. It is found that the key flow characteristics observed in the experiment are successfully reproduced by the present numerical study, namely jet induced shocks blockage, boundary layer flow separation ahead of the jet exit and shear layer vortex development along the interface between the jet and the crossflow, the latter is mainly due to the Kelvin-Helmholtz instability.
\end{abstract}

\section{Nomenclature}

$d \quad=$ jet nozzle diameter

$J \quad=$ jet-to-crossflow momentum ratio

$M \quad=$ Mach number

$P_{0} \quad=$ total pressure

Re $\quad=$ Reynolds number

$U \quad=$ streamwise velocity component

$V \quad=$ wall-normal velocity component

$U_{c} \quad=$ crossflow inlet plane velocity

$P_{c} \quad=$ crossflow inlet plane static pressure

${ }^{1}$ Lecturer, Institute of Aeronautics and Space Studies, Saad Dahleb University at Blida, Blida 09000, Algeria.

${ }^{2}$ Professor, Department of Engineering Design and Mathematics, University of the West of England, Bristol BS16 1QY, Associate Fellow AIAA. 


$\begin{array}{ll}\rho_{c} & =\text { crossflow inlet plane static density } \\ \rho_{0} & =\text { jet chamber total density } \\ \rho_{j} & =\text { jet nozzle exit static density } \\ U_{j} & =\text { jet nozzle exit velocity } \\ \delta & =\text { boundary layer thickness }\end{array}$

\section{Introduction}

$\mathrm{B}$ Ecause of the extremely short residence time of the incoming airflow in a supersonic combustive ramjet (scramjet) engine, the enhancement of supersonic flow mixing between the fuel and the air is one of most required demands in supersonic flying vehicle design. To maximize the flow mixing efficiency, it is important to achieve minimal friction losses under an environment where strong shock waves are generated and interacted with developing viscous boundary layers. Due to the high costs involved in the high-speed flight tests and the ground experiments, as well as the difficulty of developing as close as possible the test environment and making accurate measurements, numerical simulations increasingly play an important role in design and assessment of scramjet fuel injection systems and its performances.

Many scramjet fuel injection strategies have been proposed in the past decades [1], such as the use of strut, pylon, or cavities. While other studies considered basic injector type of a circular port oriented perpendicularly to the incoming free-stream flow, present work focuses on the use of injector ports that are flush with the combustor walls. This configuration has been adopted as the subject of many experimental and computational studies found in the literature. A schematic view of the jet in supersonic crossflow (JISCF), as shown in Fig. 1, highlights some salient interaction flow features observed by previous studies [2,3]. This includes a blockage of the free-stream flow induced by the transverse momentum of the jet flow, and a bow shock formed ahead of the jet exit, etc. Due to the formation of the shock-wave, and the consequent strong adverse pressure gradient, the incoming turbulent boundary layer will separate, leading to a large recirculation region. So far, majority simulations of flush-wall jet injection into crossflow use the Reynolds-averaged Navier-Stokes (RANS) turbulence modeling approaches, including predictions that use a variety of turbulence models by Uenishi et al. [4], Tam et al. [5], Palekare et al. [6], and Manna and Chakraborty [7], among others. However, it is widely accepted that for three-dimensional complex flows, turbulence models are generally incapable to capture 'real' flow physics, and thus RANS simulations can merely provide reasonable 'mean' quantities, but not the dynamic process of unsteady flow field, the latter is in fact very important and crucial in evaluating the mixing flow such as JISCF. Recently, there has been great interest in simulating flushwall injection using high-fidelity CFD simulation methods. Among them, direct numerical simulation (DNS), in which all scales of turbulence motion can be resolved, is not feasible due to prohibitively expensive computing cost for high Reynolds number flow of this type. The large-eddy simulation (LES) can resolve large-scale turbulence activities accurately and model the small-scale turbulence motions via sub-grid-scale (SGS) model. For example, Kawai and Lele [8] have used LES to study the physical aspects of the jet mixing. Ferrante et al. [9] investigated the influence of the incoming turbulent boundary layer on an inclined circular injector using LES. However, comparing to RANS, this approach is still too expensive for routinely engineering applications. As a result, Kawei and Lele [8] considered a reduced Reynolds number by a factor of six (with comparison to the reference experiment) in order to resolve the oncoming boundary layer, where Ferranti et al. [9] applied the damping to the sub-grid-scale model in the near-wall region to compensate for the limited grid resolution in the near wall region. A hybrid approach detached-eddy simulation (DES), combining RANS modeling for the near wall region and LES for regions away from wall, provides a perspective and feasible solution for high-Re engineering flows [10]. This method takes the advantages of RANS component functions as the near-wall turbulence closure, and LES of unsteady flow motions away from wall, through a flow-dependent blending function [11].

The DES approach has been successfully used in may flow types, including in particular applications where high Reynolds number is encountered such as in aerospace domain. Similar to these studies, a hybrid RANS and LES methodology will be used in this work to model the mixing flow characteristics raised from a sonic jet issuing perpendicularly into a supersonic boundary layer flow. The hybrid RANS/LES model used in this work is a derivative of detached-eddy simulation that has been specifically formulated to operate as a wall-modeled LES, this model formulation is referred as improved delayed detached-eddy simulation (IDDES) [12]. The large-scale structures of the jet plume are resolved by the LES mode of the model, and the near wall small-scale structures are modeled by the RANS mode, respectively. The simulation solver can be used for both the structured and the 

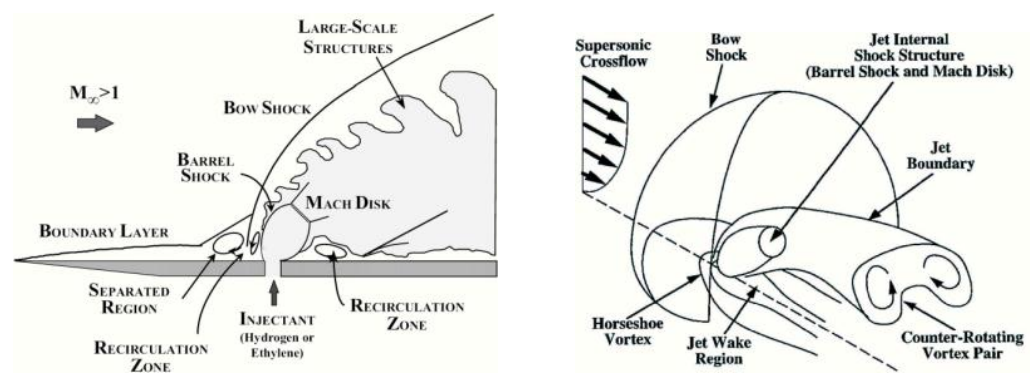

Figure 1. Schematic view of an under-expanded jet flow injecting into a supersonic crossflow $[2,3]$.

unstructured grid that provide great flexibility in grid design, and allow for efficient use of grid cells, especially when dealing with complex geometry flow problems. The time integration uses an implicit scheme, such that large time steps can be taken. The current simulation also makes use of a low-dissipation flux evaluation scheme for the spatial discretization. The simulation is able to reproduce mean wall-pressure, mean velocities components and turbulence quantities measured in experiment [16].

This present work will be focused on the assessment and the evaluation on the ability of DES approach in predicting the mixing flow properties of flush-wall injectors. The simulation results will be validated and compared with the experiment of Santiago and Dutton [13], in which a sonic air jet is issuing perpendicularly into a supersonic Mach 1.6 crossflow. Furthermore, details of turbulence closure model, numerical method, initialization procedure, turbulent inflow conditions will be described and finally simulation results especially the flow mixing characteristics will be elaborated.

\section{Simulation Set-up}

\section{A. Flow conditions}

To validate the present IDDES method, the flow conditions examined in this study are based on the experiment of Santiago and Dutton [12]. The computation uses a free-stream Mach number of $M_{\infty}=1.6$ and a Reynolds number $\mathrm{Re}_{\delta}=1.08 \times 10^{5}$, based on the free-stream conditions and the incoming boundary layer thickness at the crossflow inlet plane.

The density and pressure ratio between the nozzle chamber and crossflow are $\rho_{0 j} / \rho_{c}=5.55$ and $p_{0 j} / p_{c}=8.4$, respectively. Based on these flow conditions, the resulting jet-to-crossflow momentum flux ratio is $J=\rho_{j} u_{j}^{2} / \rho_{c} u_{c}{ }^{2}=$ 1.7. Same fluid (air) is used for both the jet and the crossflow, based on the experiment. In order to investigate the mixing between the jet fluid and the crossflow, a tracer is seeded in the jet fluid and a transport equation for a passive scalar of the jet fluid is also solved in addition to the standard Navier-Stokes equations.

\section{B. Turbulence modeling}

Turbulence is modeled using the hybrid RANS/LES approach of Shur et al. [12], which is referred to improve delayed detached-eddy simulation (IDDES). The two-equation Shear-Stress Transport (SST) turbulence model proposed by Menter [11] is used in the RANS modeling. The IDDES is an extension of the standard detached-eddy simulation (DES) formulation of Spalart et al. [14]. This standard DES formulation is now referred to as DES97.

In the DES97 formulation, the switch between the RANS mode and the LES mode is determined entirely by the grid spacing. When the grid spacing in parallel to the wall surface becomes much smaller than the boundary-layer thickness, the model switches to LES mode within the boundary-layer. This however will lower the modeled shear stresses and corrupt the RANS behavior, resulting in excessively low-friction predictions. The IDDES contains two branches that determine the RANS/LES switch. One branch is the delayed detached-eddy simulation (DDES) formulation [15]. In DDES, the switch is a function of not only the grid spacing, but of the local grid solution. If the eddy viscosity is large (at values close to that of RANS) and there are no resolved velocity fluctuations (of the LES content), the model operates in the RANS mode. In this way DDES, and hence IDDES, can maintain pure RANS behavior on grids where the wall-parallel grid spacing is much smaller than the boundary-layer thickness. This IDDES capability is useful in the scramjet combustor simulations, as combustor may contain regions of the attached boundary layers where the fuel/air mixing and combustion are not occurring. IDDES allows for these regions to be handled by RANS mode, reducing the grid resolution requirements. 
The second branch of IDDES is for situations where the grid spacing is much smaller than the boundary-layer thickness and the flow contains the LES content. Here the model operates as a wall-modeled LES (WMLES), where the RANS mode acts as a wall model for the LES mode. By modeling the near-wall region with RANS, higher Reynolds numbers flow can be simulated on a given grid relative coarse than that of a pure LES.

\section{Grid generation}

The grid used for the simulation was produced by using the commercial grid generation software Gambit, which contain a mixture of quadrilateral and tetrahedral elements. As previously mentioned, the solver used is capable of handling many elements types, such as tetrahedral, prism wedge and hexahedral elements.

The grid spacing was designed to ensure a proper resolution in all key areas of the flowfield. A cluster of highdensity cells is located along the injector wall. This area allows for the jet plume and the approaching boundary layer to be properly resolved. The grid is also stretched to the injector wall such that the first cell node is within $y^{+}<$ 5. To obtain the proper turbulence levels and the flow mixing within the jet plume, it is important to resolve the jet exit region. A close-up view of that region is shown in Fig. 2.

The coordinate system used in the simulation has its origin located at the center of the injector exit. The grid extends from $x / d=-5$ to 25 in the streamwise direction and has a length of $13 d$ in the wall normal direction $(y / d=0$ to 13). The spanwise boundaries are located at $\mathrm{z} / d= \pm 6$. The grid extends into the injector plenum chamber and it is also clustered on the injector nozzle walls. The nozzle geometry matches the experiment [13].

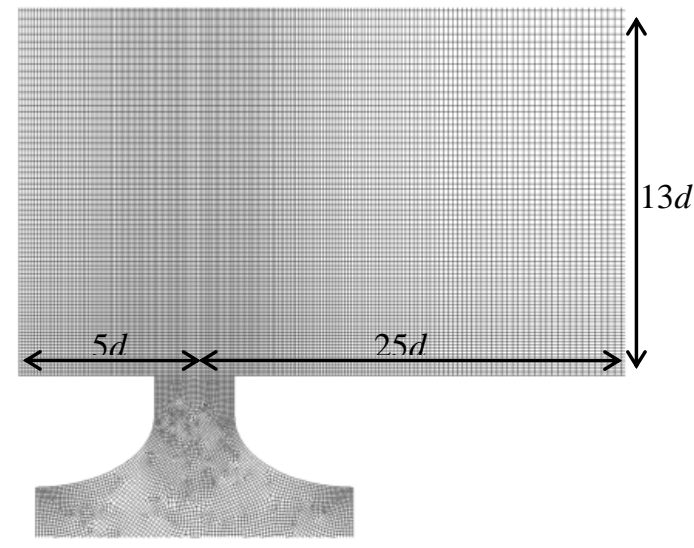

Figure 2. Computational grid shown on a cross section throughout the jet nozzle.

\section{Initial and boundary conditions}

A non-slip adiabatic wall condition is imposed for both the jet nozzle walls and the bottom wall of crossflow domain, except the jet inlet. For the computational domain, both top boundary and the side-wall boundaries are treated as free-slip walls. A standard supersonic extrapolation is performed at the crossflow domain outlet. The definition of the inlet flow boundary conditions requires extra treatments and in the present simulation, the velocity and the temperature profiles are provided from a precursor simulation of a compressible flat-plate turbulent boundary layer matching the thickness measured in the experiment at this location. For the passive scalar field, it value is set equal to one at the jet inlet boundary, whereas zero value and zero flux are imposed at the crossflow inlet plane and at all other boundaries, respectively.

The steady RANS simulation at the same flow conditions runs at first until converged achieved for providing initial flowfield, and then unsteady IDDES simulation follows till statistically converged status.

\section{Results and Discussion}

The unsteady flow simulation proceeds for a total of 100 throughflow times, defined here as the injector diameter divided by the free-stream velocity, i.e., $d / U_{\infty}$. In this time scale, the free-stream flow passes through a distance of $100 \mathrm{~d}$. This is sufficient to allow any start-up transients flow motions to exit the domain. Statistics are then accumulated for further 100 through flow times. The time step used in this work results from each through flow time taking 200 iterations, i.e. $0.005 d / U c$. This time step is greater than the explicit time step size determined by the stable criteria, demonstrating the benefit of applying the implicit time integration scheme. 


\section{A. Time-Averaged and Instantaneous Flowfield}

Comparison of the mean streamwise and the wall-normal velocity distributions between the present IDDES and the experiment [13] at the midline plane $z / d=0$ are shown in Fig. 3. The profiles are collected at four different stations downstream of the jet exit, $x / d=2, x / d=3, x / d=4$ and $x / d=5$. Similarly, profiles of velocity fluctuations are also compared to the experimental measured profiles as seen in Fig. 4. Almost the overall variation and peak amplitude of the profiles are reproduced from one realization to the next.

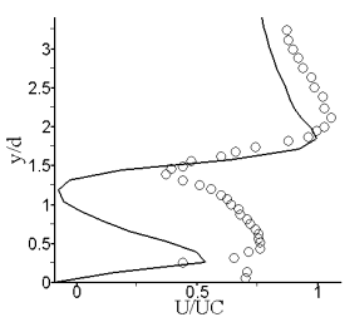

a) $x / d=2$

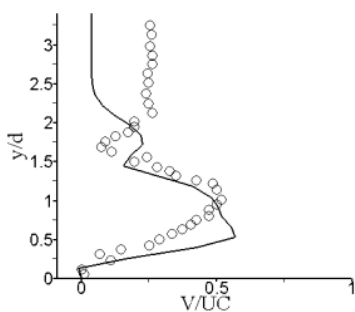

e) $x / d=2$

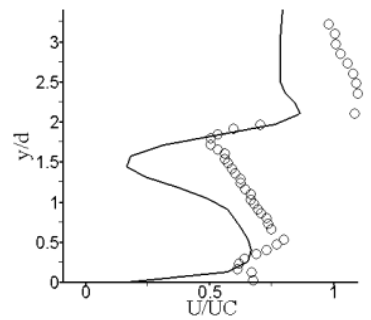

b) $x / d=3$

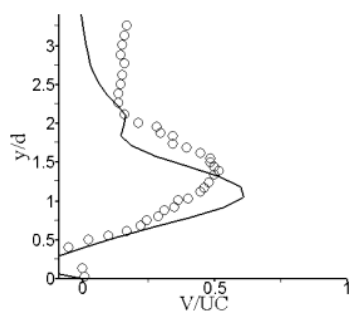

f) $x / d=3$

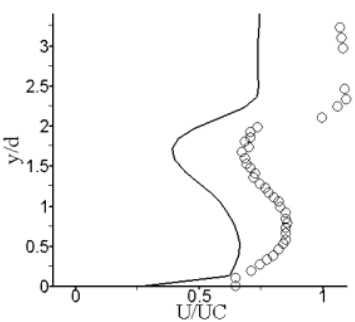

c) $x / d=4$

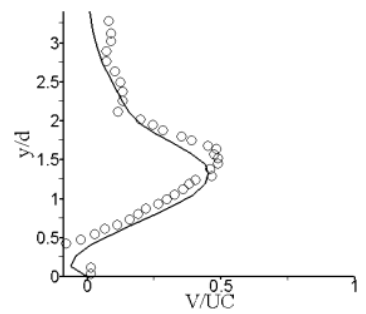

g) $x / d=4$

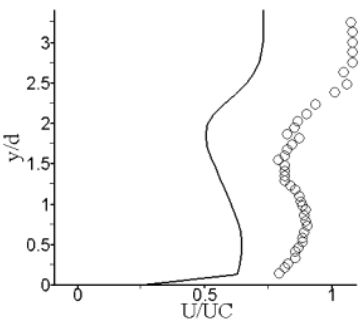

d) $x / d=5$

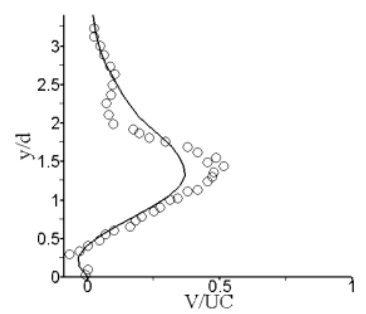

h) $x / d=5$

Figure 3. Comparison of mean streamwise $U / U_{c}$ and mean transverse $V / U_{c}$ velocity profiles between IDDES and experimental data $[13]$ at $x / d=2,3,4,5$. Solid line: IDDES, and symbol o: experiment.

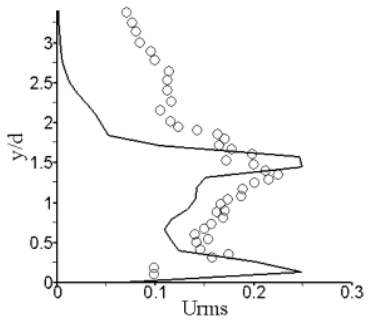

a) $x / d=2$

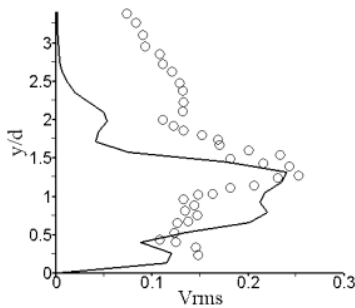

e) $x / d=2$

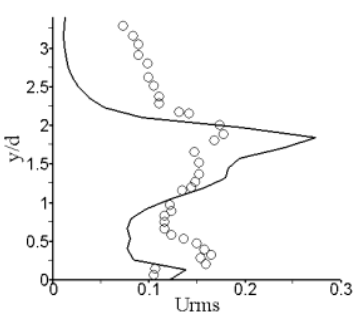

b) $x / d=3$

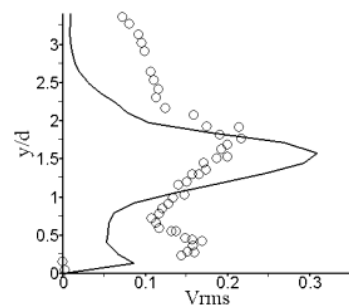

f) $x / d=3$

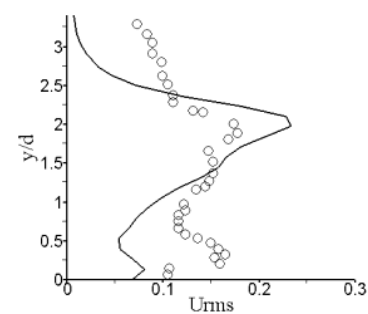

c) $x / d=4$

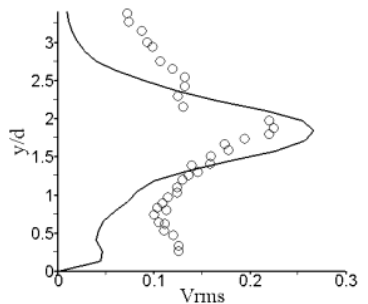

g) $x / d=4$

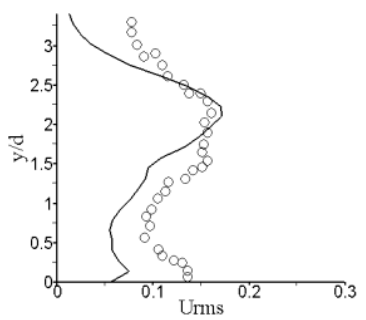

d) $x / d=5$

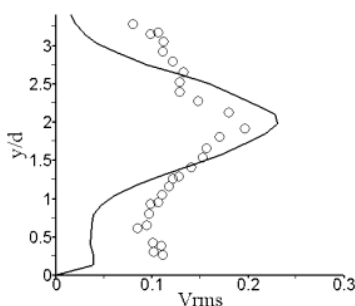

h) $x / d=5$

Figure 4. Comparison of fluctuating streamwise $U_{r m s} / U_{c}$ and fluctuating transverse $V_{r m s} / U_{c}$ velocity profiles between IDDES and experimental data [13] at $x / d=2,3,4,5$. Solid lines: IDDES, and symbols o: experiment. 
The vertical velocity profiles predicted from the IDDES calculations show a fairly good agreement with the experiment, the wake of the jet plume, shown by the maximum vertical velocity at $x / d=1.5$, is correctly captured. The peak of the vertical velocity is over-estimated at the second location, Fig. 4f, but decay quickly with downstream locations and reaches amplitudes in good agreement with the experimental data as can be seen in Fig. $4 \mathrm{~g}$ and $4 \mathrm{~h}$. The velocity fluctuations are related to the boundary layer turbulence, the wake of the jet flow and the shear vortices, and comparison is depicted in Fig. 4 . The overall profiles and the peak values amplitude of $u_{\text {rms }}$ match fairly well with the experimentally measured fluctuations. At the $x / d=3$ station a noticeable difference in the peak value is observed comparing to the experiment value. The transverse velocity fluctuations $v_{\text {rms }}$ are also overestimated in this station, but some improvement is seen for the downstream locations whilst compared to the experimental profiles. For the last station, same comments as the streamwise component are retained.

Figure 5 depicts contours of the mean streamwise and the wall-normal velocities at the middle plane, $z / d=0$. The contours are also compared to the experimental measurements [13]. In general, the mean streamwise and the wall-normal velocities fields predicted by the present IDDES calculations are qualitatively in good agreement with the experiment. However, the IDDES predicted flow fields has also show some exceptional strengths of the flow velocity in the separated flow region, immediately downstream of the jet injection and also downstream of the flow reattachment.

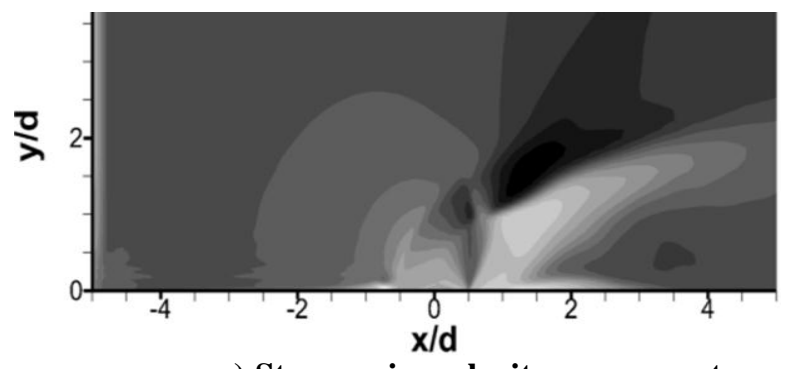

a) Streamwise velocity component

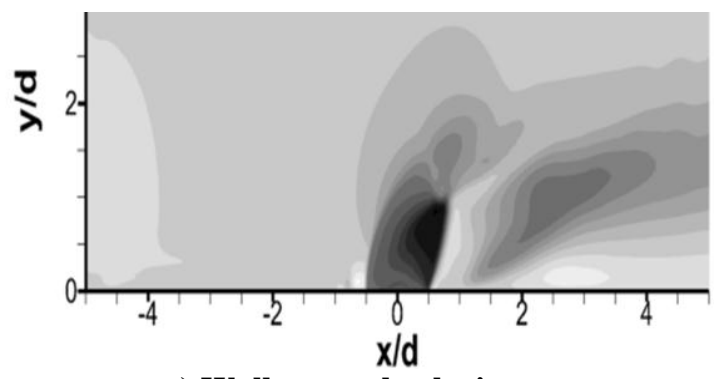

c) Wall-normal velocity component

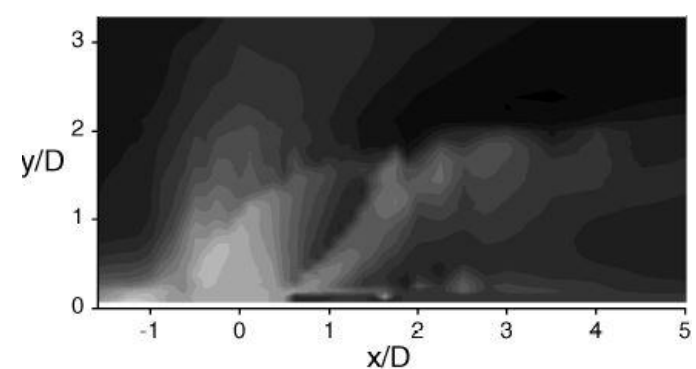

b) Experiment

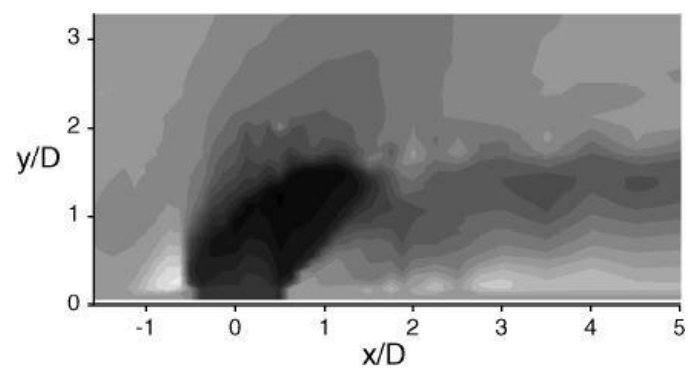

d) Experiment

Figure 5. Comparison of mean streamwise (top) and wall-normal (bottom) velocity contours between IDDES and experiment [13] at $z / d=0$.

The instantaneous velocity contours at two successive cross-section stations $x / d=3$ and $x / d=5$ downstream of the injector, are shown in Fig. 6. The kidney-shaped Counter Rotating Vortex Pair (CRVP) is clearly observed in these plots. While the overall shape is correctly captured by the numerical simulation, the width of the mixing region, defined by either $u_{r m s}$ or $v_{r m s}$ is however, under-estimated as proved by the under-estimated peaks in Fig. $4 \mathrm{~b}$ and Fig. 4f. While at the second location $x / d=5$, the size of the mixing area is fairly good as can be demonstrated by the line plot of these variables in Fig. $4 \mathrm{~d}$ and Fig. $4 \mathrm{~h}$. 


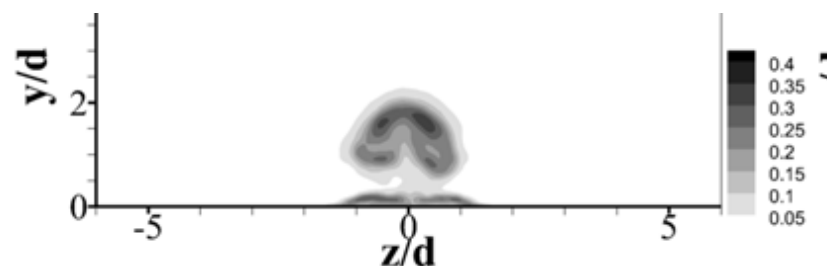

a) $u_{r m s}$ at $x / d=3$

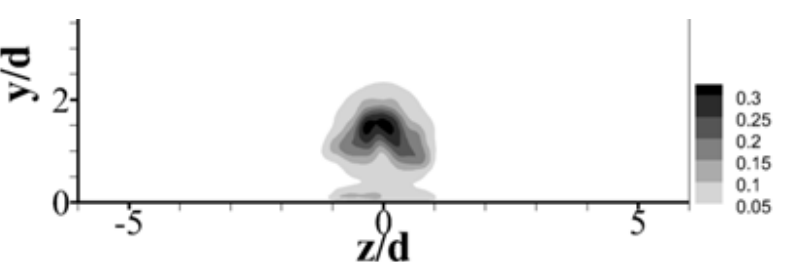

b) $v_{r m s}$ at $x / d=3$

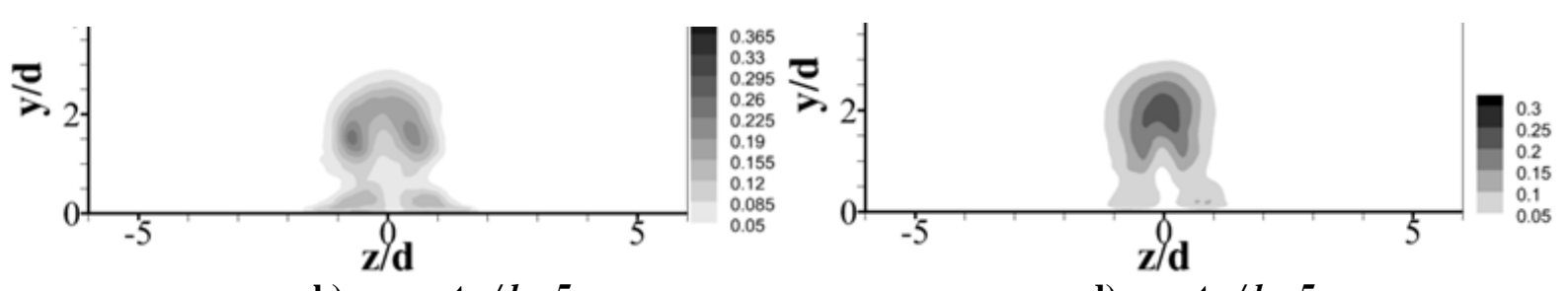

b) $u_{r m s}$ at $x / d=5$

d) $v_{r m s}$ at $x / d=5$

Figure 6. Contours of streamwise and wall-normal fluctuations in two downstream cross-planes of the jet exit $(x / d=3$ and $x / d=5)$.

Figure 7 compares the predicted mean wall-pressure profiles to the experimental data qualitatively at two spanwise stations $\mathrm{z} / d=1$ and 2 . It can be seen that the IDDES predicts the right trend and variation compare to the experimental measurements with maximum discrepancy about a factor of 2 . Indeed, there are some qualitative agreements in terms of a few flow features, including the pressure rise upstream of the injection due to the separation bow shock, relative low-pressure region downstream of the first peak, due to the horseshoe vortex. The second pressure rise downstream of the jet exit could be induced by the wake vortex and there is large low-pressure region observed further downstream of the jet exit.

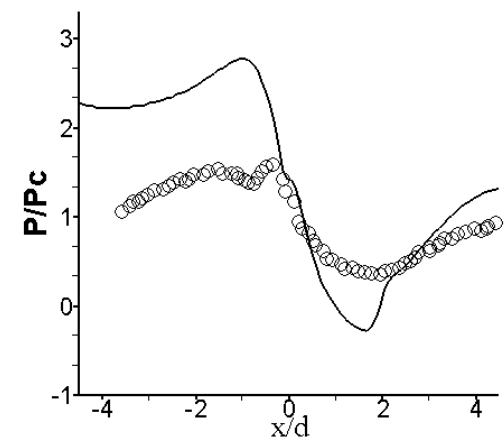

a) $z / d=1$

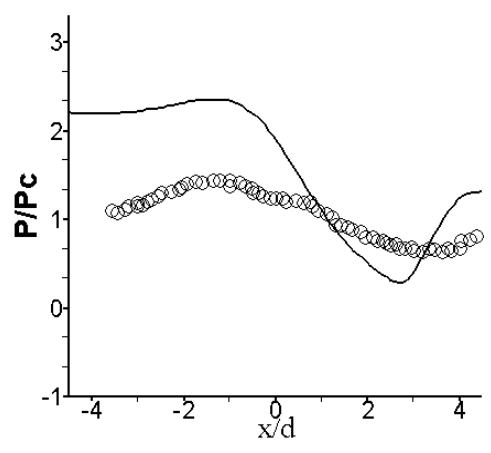

b) $z / d=2$

Figure 7. Comparaison of mean wall-pressure $p / p_{c}$ distributions between IDDES and experimental PSP measurment [16] at $z / d=1$ and 2, Solide lines: IDDES and symbols o: experiment.

In order to obtain further insights on the flow field distributions in the near-wall, a plane-view at the wall surface, $i, e$., the $X Y$ plane at $y / d=0$ of the mean pressure distributions is depicted in Fig. 8, where low and high pressure regions can be seen clearly. Upstream of the jet injection, the bow shock induced by the jet blockage causes an increase of the surface pressure, downstream of the jet injection the detected Prandtl-Mayer expansion fan which enables the undernearths sonic jet flow to entering the crossflow envirenment, by accelerating the flow stream and the therfore a decrease of flow pressure as clearly shown downstream of the jet injection. 


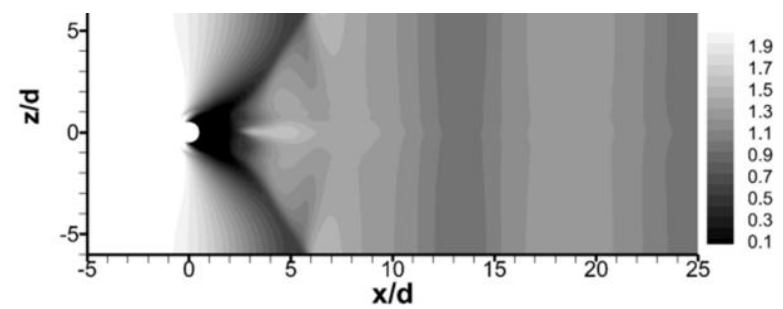

Figure 8. The contours of mean wall-pressure $p / p_{c}$.

\section{B. Time-Averaged and Instantaneous Passive Scalar Field}

Mean passive scalar field (jet fluid) distributions at the midline plane $z / d=0$ at downstream stations $x / d=2,3,4$, 5 obtained by the present IDDES calculations with comparison to LES predictions of the same flow configuration and conditions performed by Kawai and Lele [8] are shown in Fig. 9. Overally a good agreement to the LES calculations is observed in term of reproduction of peak values from these two simulation approachs. By inspection of the maximum values positions depicted by these lines, these peak positions in the transverse direction (i.e. $y$-axis), correspond to higher jet fluid concentration around the cores of the counter-rotating jet vortices (CRVP) as can be observed when investigating the Fig. 10.

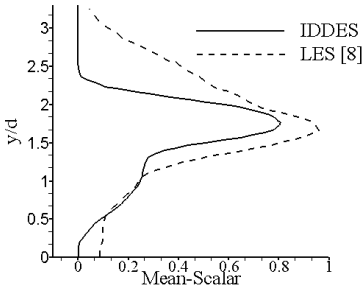

a) $x / d=2$

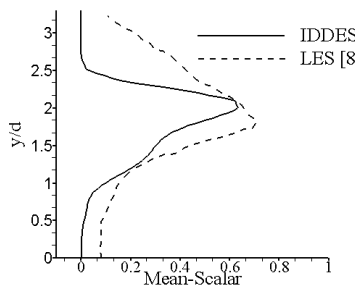

b) $x / d=3$

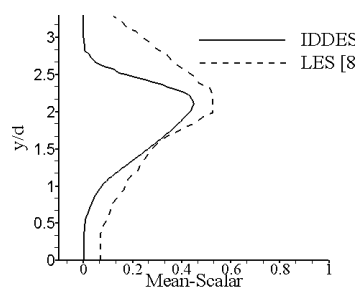

c) $x / d=4$

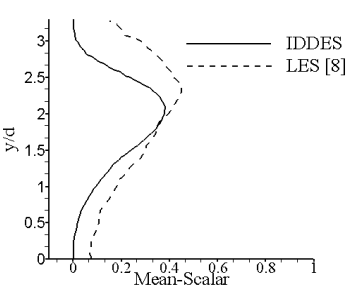

d) $x / d=5$

Figure 9. Comparison of mean passive scalar of jet fluid distributions at middle plane $z / d=0$, at downstream stations $x / d=2,3,4$, and 5, Solid lines: IDDES and Dashed lines: LES [8].

Figure 10 shows the mean passive scalar (of the jet fluid) and its fluctuations at three successive cross-section planes $x / d=1,3$ and 5. It is clear that, the mixing of the jet fluid along the windward jet boundary is evident by enhanced entrainment caused by caused by the CRVP. In downstream locations, the large-scale vortex structure (i.e. CRVP) breaks down to the finer structures, possibly due to K-H instability. This indicates the importance of the turbulent structures that determine the behavior of the jet fluid stirring and subsequent mixing along its projectile path. These observations are supported when the 'rms' value ( $c f$ Fig. 12) of the jet fluid tracer fluctuations are investigated. The high intensity region in the 'rms' value of the jet fluctuations spreads over a much wider region compared to that of the mean scalar. There is some larger extent of the high 'rms' value that might indicate the progressive mixing process of the jet fluid with the crossflow.

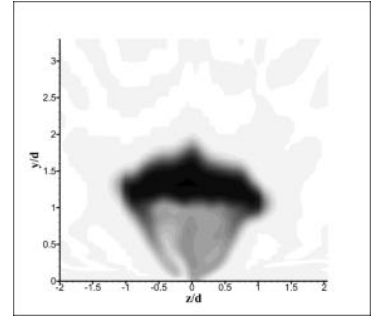

a) $x / d=1$

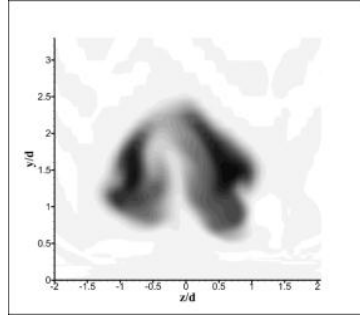

b) $x / d=3$

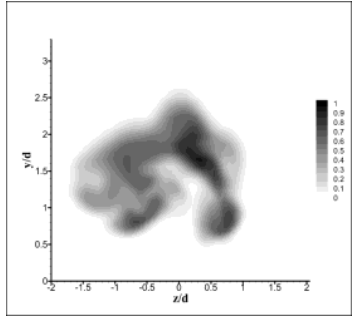

c) $x / d=5$

Figure 10. Mean passive scalar distributions at three cross-view planes $x / d=1,3$ and 5 . 
Figure 11 further illustrate jet fluid mixing by tracing its profile evolution along wall-normal lines at downstream locations $x / d=2,3,4$, and 5. The results also compare with the reference LES predicted profiles [8], where good agreement is observed between the IDDES and the LES predictions at some extent. Figure 12 shows contours of the jet fluid fluctuation (rms) distributions, and the results obtained support the earlier observations about the high intensity region of rms values discussed in the previous paragraph.

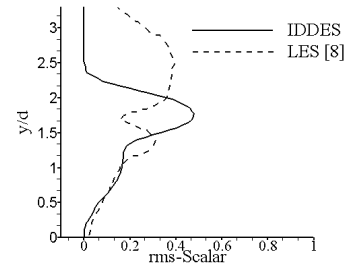

a) $x / d=2$

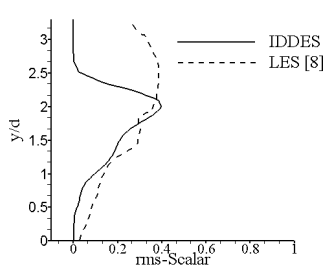

b) $x / d=3$

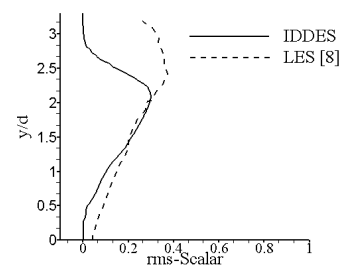

c) $x / d=4$

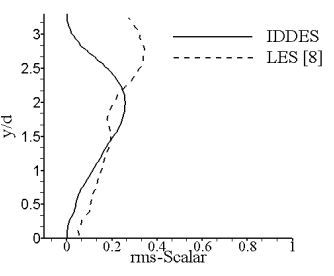

d) $x / d=5$

Figure 11. Comparison of rms jet fluid tracer fluctuations distributions at middle plane $z / d=0$, at downstream stations $x / d=2,3,4$, and 5, Solid lines: IDDES and Dashed lines: LES [8].

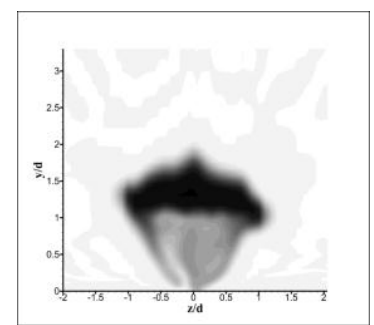

d) $x / d=1$

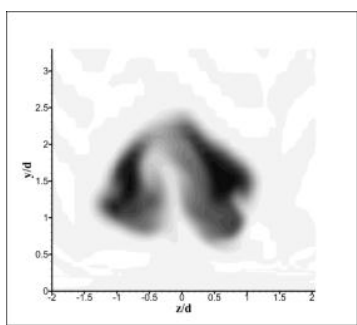

d) $x / d=3$

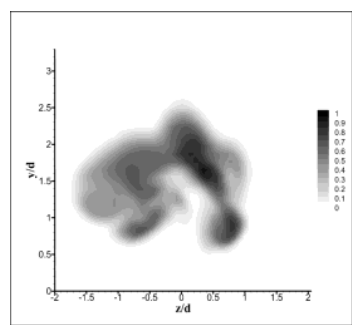

e) $x / d=5$

Figure 12. The jet fluid fluctuations (rms) of distributions at three cross-view planes $x / d=1,3$ and 5 .

A slice of the passive scalar field is shown in Fig. 13. The slice correspond to the middle plane $X Y$ at $z / d=0$. Note that the scalar value varies smoothly from zero (black) to one (white). The barrel shock is clearly seen in the figure. After that, in downstream locations, the Kelvin-Helmholtz vortices that form from the windward side of the jet 'plume tube' can be seen in the figure. As a result, the jet fluid is progressively stirred with the crossflow, entrained into the main crossflow, and subsequently flow mixing is enhanced.

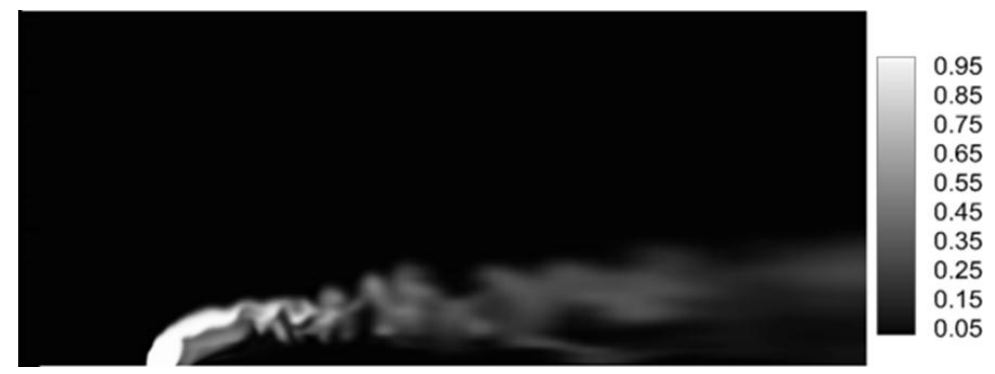

Figure 13. Contour of instantaneous jets fluid tracer value at the center plane $z / d=0$.

The 3D features of captured vortex structures are visualized by the instantaneous iso-surfaces of the second invariant of velocity gradient tensor $Q$, as shown in Fig. 14. It can be seen that the jet is elongated along the relatively large-scale longitudinal vortex structures. In the meantime, the horseshoe vortex formation in the near wall upstream of the jet injection is also clearly seen in Fig. 14, together with the hanging vortices formed in the near 
wake field region. Finally, the windward quasi-ring like vortices resulting from the K-H instability are also visualized. As can be seen in this figure, these large roller vortices structures are convected downstream, graduately weaken and broken down following the interactions with the CRVP developed in the turbulent flow region. This indicates the importance of these eddy structures that will determine the behavior of the jet fluid stirring and subsequent mixing.

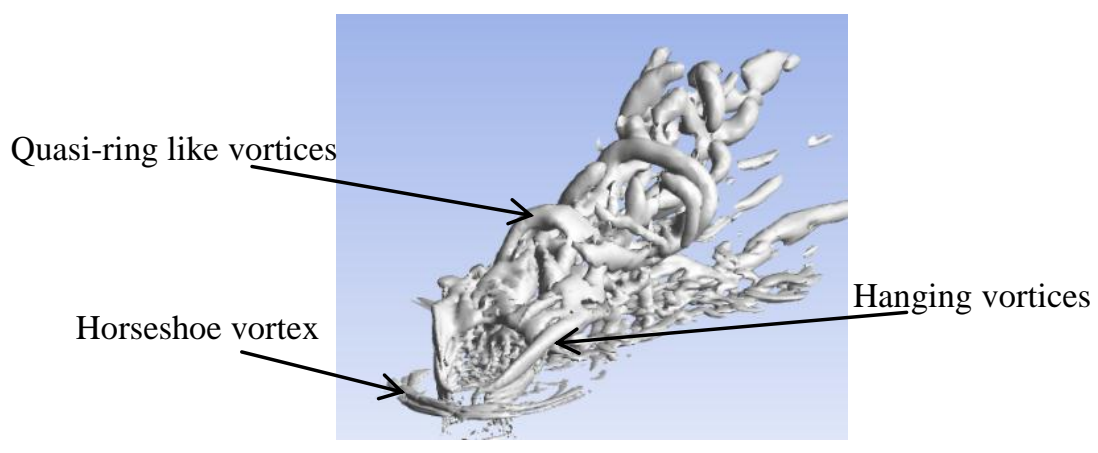

Figure 14. Isosurface of the second invariant of velocity gradient tensor, $Q=10^{9} \mathrm{~s}^{-2}$.

\section{Conclusion}

The DES simulation results of the mixing flow characteristics observed in a sonic jet injected perpendicularly into a supersonic Mach 1.6 crossflow has been presented. The computation uses a low-dissipation flux evaluation method and fully implicit time integration scheme on a block-structured grid framework. Turbulence is modeled using a hybrid RANS/LES methodology. Using this approach allows for the large-scale turbulent flow structures of the flowfield to be properly resolved by the LES mode, while the small-scale turbulent flow structures in the near wall region to be modelled by the RANS mode. This combination provides a more realistic approximation to the instantaneous flow mixing process as investigated in this study, than that by steady-state simulations.

The validation of this this approach has been carried out for simulating mixing process associated with a sonic injection into a supersonic crossflow, which can be found in the in scramjet combustor section. The results have shown some reasonable agreements when compared to experimental measurements in terms of mean and standard deviation flow field. Both the mean and fluctuating flow variables are compared against the available test data at the near jet exit region and the downstream locations up to five jet diameter positions. In general, most key flow features of this flow configuration such as shock systems, large-scale vortices are reproduced successfully.

\section{Acknowledgments}

This study was conducted at the University of the West of England Bristol, UK. The first author would also like to thank the Ministry of Higher Education and Scientific Research, Algeria through the University of Blida 1 for the funding support of this research project.

\section{References}

${ }^{1}$ Curran, E.T., and Murthy, S.N.B. (eds.), "Scramjet Propulsion," Progress in Astronautics and Aeronautics, Vol. 189, AIAA, Reston VA, 2000.

${ }^{2}$ Ben-Yakar, A., Mungal, G.M. and Hanson, R.K., "Time evolution and mixing characteristics of hydrogen and ethylene transvers jet in supersonic crossflows," Physics of Fluids, Vol. 18, 026101, 2006.

${ }^{3}$ Gruber, M.R., Nejad, A.S., Chen, T.H., and Dutton, J.C., "Mixing and penetration studies of sonic jets in a Mach 2 freestream," J. of Propulsion and Power, Vol. 11, No. 2, 1995, pp. 315-323.

${ }^{4}$ Uenishi, K., Roger, R.C., and Northram, G.B., "Numerical Predictions of a Rearward-Facing step Flow in a Supersonic Combustor," J. of Propulsion and Power, Vol. 5, No. 2, 1989, pp. 158-164.

${ }^{5}$ Tam, C., Baurle, R.A., and Gruber, M.R., "Numerical Study of Injection into a Supersonic Crossflow," AIAA Paper 992254, June 1999. 
${ }^{6}$ Palekar, A., Truman, C.R., and Vorobieff, P., "Prediction of Transverse Injection of a sonic Jet in Supersonic Crossflow," AIAA Paper 2005-5366, June 2005.

${ }^{7}$ Manna, P., and Chakraborty, D., "Numerical Investigation of Transverse Sonic Injection in a Non-Reacting Supersonic Combustor," J. of Aerospace Engineering, Vol. 219, No.32, 2005, pp. 205-215.

${ }^{8}$ Kawei, S., and Lele, S.K., "Large-Eddy Simulation of Jet Mixing in Supersonic Crossflow," AIAA Journal, Vol. 48, No. 9, 2010, pp. 2063-2083.

${ }^{9}$ Ferrant, A., Matheou, G., and Dimotakis, P.E., "LES of Inclined Sonic Jet into a Turbulent Crossflow at Mach 3.6," Journal of Turbulence, Vol. 12, No. 2, 2011, pp. 1-32.

${ }^{10}$ Spalart, P.R., Jou, W.H., Strelets, M.K., and Allmaras, S.R., "Comments on the feasibility of LES for wings, and on hybrid RANS/LES approach," In the first AFOSR International Conference on DNS/LES, Ruston, Louisiana, 1997.

${ }^{11}$ Menter, F.R., "Two-equation Eddy-viscosity Turbulence Models for Engineering Applications," AIAA Journal, Vol. 8, No. 32, 1194, pp. 1598-1605.

${ }^{12}$ Shur, M.L., Spalart, P.R., Strelets, M.K., and Tarvin, A.K., "A Hybrid RANS-LES Approach with Delayed-DES and Wall Modeld LES Capabilities," International Journal of Heat and Fluid Flow, Vol. 29, No. 6, 2008, pp. 1638-1649.

${ }^{13}$ Santiago, J.G., and Dutton, J.C., "Velocity Measurements of a Jet Injected into a Supersonic Crossflow," Journal of Propulsion and Power, Vol. 13, No. 2, 1997, pp. 264-273.

${ }^{14}$ Spalart, P.R., Jou, W-H., Strelets, M.K., and Allmaras, S.R., "Comments on the Feasibility of LES for Wings and on Hybrid RANS/LES Approach," Advances, in DNS/LES, $1^{\text {st }}$ AFSOR International Conference on DNS/LES, Greyden, Columbus, OH, Aug. 4-8, 1997.

${ }^{15}$ Spalart, P.R., Deck, S., Shur, M.L., Squires, K.D., Strelets, M.K., and Travin, A.K., “A New version of Detached-Eddy Simulation, Resistant to Ambiguous Densities", Theoretical and Computational Fluid Dynamics, Vol. 20, 2006, pp. 181-195.

${ }^{16}$ Everett, D.E., Woodmansee, M.A., Dutton, J.C., and Morris, M.J., "Wall Pressure Measurements for a Sonic Injected Transversely Into a Supersonic Crossflow,” Journal of Propulsion and Power, Vol. 14, No. 6, 1998, pp. 861-868. 